\title{
Caenorhabditis elegans VEM-1, a Novel Membrane Protein, Regulates the Guidance of Ventral Nerve Cord-Associated Axons
}

\author{
Erik Runko $^{1}$ and Zaven Kaprielian ${ }^{1,2}$ \\ Departments of ${ }^{1}$ Neuroscience and 2 Pathology, Albert Einstein College of Medicine, Bronx, New York 10461
}

In the developing CNS, pathfinding growth cones use intermediate target- and pioneer axon-associated guidance cues to navigate along stereotypical trajectories. We previously showed that the novel membrane-associated protein Vema is localized to the floor plate and the optic chiasm, intermediate targets located at the ventral midline of the spinal cord and diencephalon in the developing rodent CNS, respectively. Here, we report that the Caenorhabditis elegans ortholog of vema, vem-1, is expressed by the AVG pioneer midline neuron and by several neurons that extend longitudinally projecting axons into the ventral nerve cord (VNC). In vem-1 mutants and vem-1(RNAi) animals, a subset of posteriorly projecting interneuron axons either fail to extend ventrally to the VNC and, instead, assume aberrant lateral positions or are inappropriately located in the left tract of the VNC. In addition, ventral motor neuron axons exhibit pathfinding errors within the VNC and along the dorsoventral body axis. The conserved UNC-40/DCC and SAX-3-/Robo receptors mediate signaling events that regulate axon guidance in a wide variety of systems. Double-mutant analyses reveal that vem-1 genetically interacts with unc-40 and is likely to function in parallel with sax-3 to regulate the guidance of a subset of VNC-associated interneuron and motor neuron axons. Consistent with these genetic data, we also show that VEM-1 is capable of physically interacting with UNC-40 but not SAX-3.

Key words: vema; VEM-1; AVG; VNC; pioneer; glr-1; unc-40

\section{Introduction}

Growing axons travel significant distances along stereotypical trajectories to reach their final targets. Intermediate targets divide these trajectories into a series of short segments and provide axons with the guidance cues required to extend along the next leg of their journey (Tessier-Lavigne and Goodman, 1996; Dickson, 2002). In many model systems, early developing pioneer axons represent additional sources of guidance information (TessierLavigne and Goodman, 1996).

The Caenorhabditis elegans ventral nerve cord (VNC) is an asymmetric structure comprising two parallel, longitudinally ori-

\footnotetext{
Received May 13, 2004; revised Aug. 26, 2004; accepted Aug. 26, 2004.

This work was supported by a predoctoral training grant (T32-NS07098) from the National Institute of Neurological Disorders and Stroke and a National Institutes of Health First Independent Research Support and Transition award (R29-NS34847), as well as grants from the Whitehall Foundation (F98-03) and the March of Dimes (FY02185) to Z.K. We thank Oliver Hobert, Bill Wadsworth, Harald Hutter, Bruce Wightman, David Miller, Piali Sengupta, Yishi Jin, Joseph Culotti, and Theresa Stiernagle of the Caenorhabditis Genetics Center (University of Minnesota, Minneapolis, MN) for providing strains, and Andrew Fire, Dave Pilgrim, Monica Driscoll, and Yuji Kohara for plasmid vectors and CDNA clones. Barbara Perry and Paul Sternberg provided the deletion library and allowed one of us (E.R.) to perform the screening in the Sternberg laboratory at the California Institute of Technology (Pasadena, CA), and Cornelia Bargmann provided the GST fusion protein constructs. Haftan Eckholdt provided statistical analysis. We also thank Oliver Hobert, Hannes Bülow, Dave Hall, Scott Emmons, Andrew Hahn, Robyn Lints, and Doug Portman for helpful suggestions and technical advice, as well as Alex Runko, Oliver Hobert, and Hannes Bülow for critical reading of this manuscript.

Correspondence should be addressed to Zaven Kaprielian, Departments of Neuroscience and Pathology, Albert Einstein College of Medicine, Kennedy Center, Room 624, 1410 Pelham Parkway South, Bronx, NY 10461. E-mail: kapriel@aecom.yu.edu.

E. Runko's present address: College of Physicians and Surgeons, Department of Biochemistry and Molecular Biophysics, Columbia University, Hammer Health Sciences Center 724, 701 West 168th Street, New York, NY 10032. DOl:10.1523/JNEUROSCI.2385-04.2004

Copyright $\odot 2004$ Society for Neuroscience $\quad 0270-6474 / 04 / 249015-12 \$ 15.00 / 0$
}

ented axon tracts located on either side of the ventral midline (White et al., 1976, 1986). The AVG neuron extends an axon that pioneers the right tract of the VNC (Durbin, 1987; Wightman et al., 1997), whereas the PVPR and PVT axons pioneer the left and right tracts of the VNC (Durbin, 1987; Wightman et al., 1997; Aurelio et al., 2002), respectively. Animals that lack AVG (Durbin, 1987; Hutter, 2003), PVPR (Durbin, 1987) or PVT (Ren et al., 1999; Aurelio et al., 2002) display a disorganized VNC; some axons are inappropriately located in the left or right tract. Many early developing VNC-associated motor neurons extend axons that grow circumferentially toward the dorsal nerve cord (DNC) along the right side of the animal (White et al., 1986; Durbin, 1987; Huang et al., 2002; Hobert and Bulow, 2003; Hutter, 2003). In AVG-ablated animals, VNC-associated D-type motor neuron axons inappropriately extend along the left side of the animal (Durbin, 1987; Hutter, 2003).

Evolutionary conserved guidance systems participate in the formation of the C. elegans VNC (Chisholm and Tessier-Lavigne, 1999; Hobert and Bulow, 2003). Most notably, UNC-6/netrin and its receptors, UNC-40/DCC and UNC-5, mediate long-range pathfinding toward, or away from, the midline, respectively $(\mathrm{Cu}-$ lotti and Merz, 1998; Dickson, 2002; Wadsworth, 2002). In addition, local interactions between the SLT-1/Slit ligand and its SAX3/Robo receptor appear to segregate axons to the left or right tract of the VNC (Zallen et al., 1998, 1999; Hao et al., 2001).

Heteromeric complexes of guidance receptors, as well as proteins that regulate the availability of guidance receptors on the cell surface, modulate the response of axon/growth cones to a 
variety of environmental cues (Yu and Bargmann, 2001; Dickson, 2002; Grunwald and Klein, 2002). As a striking example of the latter mechanism, Drosophila Commissureless (Comm) (Tear et al., 1996; Kidd et al., 1998) functions as a sorting receptor for Robo that facilitates midline crossing by selectively rendering precrossing axons insensitive to the midline-associated repellent Slit (Keleman et al., 2002; Myat et al., 2002).

Vema is a novel membrane-associated protein that is localized to midline-associated intermediate targets in the developing rodent CNS (Zhu et al., 1998; Runko et al., 1999; Runko and Kaprielian, 2002). We show here that a C. elegans ortholog of vema, vem-1, is expressed in the AVG pioneer neuron and a subset of axons that project into the VNC. A vem-1 null mutant displays defects in the patterning of VNC-associated interneuron and motor neuron axons. Genetic and biochemical data suggest that vem-1/VEM-1 may operate together with unc-40/UNC-40.

\section{Materials and Methods}

Strains. C. elegans were grown and maintained using standard methods (Brenner, 1974). The C. elegans Bristol strain N2 was used as the wildtype strain, and pha-1(e2123ts), unc-40(e271), unc-40(e1430), sax3(ky123), and slt-1(eh15) mutants were provided by the Caenorhabditis Genetics Center (University of Minnesota, Minneapolis, MN). GFP (green fluorescent protein) reporter strains used in this study include the following: VH15 (rhIs4 [glr-1::GFP; $d p y-20(+)]$ III; obtained from Harald Hutter, Max Planck Institute, Munich, Germany), edIs20 [unc-119::GFP; rol-6 (su1006); obtained from Bill Wadsworth, University of Medicine and Dentistry of New Jersey, Newark, NJ], SK4005 [zdIs5 ( mec-4::GFP) I; lin-15(+); obtained from Scott Clark, Skirball Institute, New York University, New York, New York], oyIs14 [sra-6::GFP reporter; lin-15(+) (Troemel et al., 1995); obtained from P. Sengupta, Brandeis University, Waltham, MA], juIs76 (unc-25::GFP reporter; obtained from Yishi Jin, University of California, Santa Cruz, CA), evIs $82 b$ [unc-129:: GFP reporter (Colavita et al., 1998); obtained from J. Culotti, Mount Sinai Hospital, Toronto, Ontario, Canada], bwIs2 ( $f l p-1:: G F P$ reporter; obtained from B. Wightman, Muhlenberg College, Allentown, PA), ns106 [lin-11::GFP reporter (Reddien et al., 2001)], wdIs3 [unc-4::GFP reporter (Miller and Niemeyer, 1995); obtained from D. Miller, Vanderbilt University, Nashville, TN], otIs39 [unc-47::GFP reporter; lin-15(+) (Aurelio et al., 2002); obtained from O. Hobert, Columbia University, New York, NY], otIs33 (kal-1::GFP reporter; obtained from O. Hobert), mgIs18 [ttx-3::GFP reporter (Altun-Gultekin et al., 2001); obtained from O. Hobert], and otEx1082 [inx-18::GFP reporter (Bulow et al., 2004); obtained from O. Hobert].

Northern analysis and $5^{\prime}$ rapid amplification of cDNA ends. Total RNA derived from mixed-stage C. elegans larvae was isolated using TRI REAGENT (Molecular Research Center, Cincinnati, $\mathrm{OH}$ ) according to the instructions of the manufacturer. A total of $20 \mu \mathrm{g}$ of RNA was loaded into each lane and separated on a 1.5\% agarose-formaldehyde gel, transferred to a nylon membrane, and UV cross-linked as described previously (Kaprielian et al., 1995). A vem-1 PCR product that encodes exons 1 and 2 was gel purified, ${ }^{32} \mathrm{P}$-labeled (random primed labeling kit; Boehringer Mannheim, Indianapolis, IN), and used as a probe, all as described previously (Kaprielian et al., 1995).

The 5' end of vem-1 mRNA was analyzed using the RLM-RACE (RNA ligase-rapid amplification of cDNA ends) kit (Ambion, Austin, TX). Sequencing was performed with vem-1-specific primers and primers corresponding to the SL1 and SL2 trans-splice leader sequences.

vem- 1 expression analysis. To generate the transcriptional vem-1::GFP fusion, upstream vem-1 sequence (1955 bp; nucleotides 10552-8597; numbering refers to position on cosmid K07E3) was amplified by the PCR, and the product was fused to GFP (coding region of the pPD95-79 vector, kindly provided by Andy Fire, Stanford University, Stanford, CA) (Hobert et al., 1999). Transgenic lines were created by injecting the vem-1::GFP reporter at $1 \mathrm{ng} / \mu \mathrm{l}$ into a pha-1(e2123ts); him-5(e1490) mutant background using $\mathrm{pBX}$ ( pha-1 wild-type expression construct) at $175 \mathrm{ng} / \mu \mathrm{l}$ to rescue pha-1 larval lethality (Granato et al., 1994). Micro- injections of young adult C. elegans hermaphrodites were performed as described previously (Mello and Fire, 1995). Several independent transgenic lines displaying the same labeling pattern were produced, and integrated reporter lines were generated by gamma irradiation. The chromosomally integrated array rzIs $1=$ integrated $E x(p v e m-1:: G F P ; p B X)$; pha-1(e2123ts); him-5(e1490) was used for the expression analysis. Nematodes were mounted on a slide in a small drop of M9 buffer on a $\%$ agarose pad supplemented with 1\% 1-phenoxy-2-propanol (paralytic agent) and analyzed by epifluorescence microscopy.

A translational VEM-1::GFP fusion was constructed by amplifying upstream vem-1 sequence, as well as the vem-1 coding locus minus the stop codon (3595 bp; nucleotides 10552-6957; numbering refers to position on cosmid K07E3) by the PCR, and fusing the product in frame to GFP as indicated above. Transgenic lines were created by injecting the VEM-1::GFP reporter at $2.4 \mathrm{ng} / \mu \mathrm{l}$ and examined as indicated above, except that extrachromosomal array lines were used for the expression analysis.

Isolation of a vem-1 mutant. The mutant allele vem-1(rz1) was isolated by screening a UV-trimethylpsoralen-induced C. elegans deletion library, representing $\sim 130,000$ mutagenized genomes, using a PCR-based sib selection procedure (Jansen et al., 1997) in the Sternberg Laboratory at California Institute of Technology (Pasadena, CA). Specifically, we used two pairs of nested primers that ultimately amplify $2140 \mathrm{bp}$ of the vem-1 gene. The first round of the PCR was performed with an "outside" primer pair (6757F and 9179R) that yielded a 2422 bp product, and the second round was performed with an "inner" primer pair $(6838 \mathrm{~F}$ and $8978 \mathrm{R}$ ) that yielded a 2140 bp product, using wild-type genomic DNA (numbering refers to positions within the K07E3 cosmid). Worms harboring the vem-1 deletion mutant vem-1(rzl) were isolated and backcrossed against wild-type (N2) animals five times. To identify the lesion in the vem-1(rz1) mutant allele, genomic DNA was prepared and sequenced using standard techniques. The vem-1(rzl) mutant animal harbors a 660 nucleotide deletion that spans nucleotides 8087-7427 (numbering refers to positions within the K07E3 cosmid) and that removes exon 1 and all of exon 2, except for the last $803^{\prime}$ nucleotides.

RNA interference. DNA corresponding to the regions of the vem-1 gene containing exons $1-2$ (and intervening introns) were prepared and cloned into pBluescript (Stratagene, La Jolla, CA) as described previously (Fire et al., 1998). Sense and antisense RNA were prepared using either T3 or T7 polymerases, and the reactions were then pooled, incubated to promote annealing, and examined-quantified by agarose gel electrophoresis as described previously (Fire et al., 1998). Subsequently, the double-stranded RNA (dsRNA) or buffer alone (control) was injected into the gonads of the various transgenic lines, and phenotypes were assessed by epifluorescence microscopy in L3/4 progeny.

For heat-shock-induced dsRNA, a genomic fragment containing exons 1-2 (and intervening introns) of vem-1 was first amplified by two separate PCRs, using primers that introduce unique restriction sites at each end of these fragments, and subsequently cloned into pCRII (Invitrogen, San Diego, CA). Restriction digests were performed to isolate DNA fragments harboring the genomic vem-1 sequence (exons 1-2) in both orientations, and a three-way ligation was performed to insert the inverted repeat into the calf intestinal alkaline phosphatase-treated $C$. elegans expression vector $\mathrm{pPD} 49.78$, containing the $h s p 16-2$ promoter and the $3^{\prime}$ untranslated region of muscle myosin unc-54 (Mello and Fire, 1995), all as described previously (Tavernarakis et al., 2000). This construct or pPD49.78 alone (control) was then transformed into the glr-1::GFP and vem-1::GFP reporter lines. To examine the effects of vem-1 hairpin dsRNA, individual worms were heat shocked for $4 \mathrm{hr}$ at $35^{\circ} \mathrm{C}$ and returned to $20^{\circ} \mathrm{C}$, and the $\mathrm{L} 3 / 4$ progeny were collected and scored.

vem-1 construct generation. A 1936 bp HindIII/ApaI fragment of cosmid K07E3 was injected at $33 \mathrm{ng} / \mu \mathrm{l}$ with the dominant transformation marker pRF4 rol-6(su1006) at $113 \mathrm{ng} / \mu$ l into vem-1(rz1);rhIs4 animals. The VEM-1::GFP translational reporter was injected at $2.4 \mathrm{ng} / \mu \mathrm{l}$ with pRF4 rol-6(su1006) at $50 \mathrm{ng} / \mu \mathrm{l}$ into vem-1(rz1);rhIs4 or vem-1(rz1); juIs76 animals. Injection of only pRF4 rol-6(su1006) at $113 \mathrm{ng} / \mu \mathrm{l}$ into vem-1(rz1);rhIs4 animals was used as the control.

The $g l r-1 p:: v e m-1$ construct was generated by ligating a PCR-amplified 


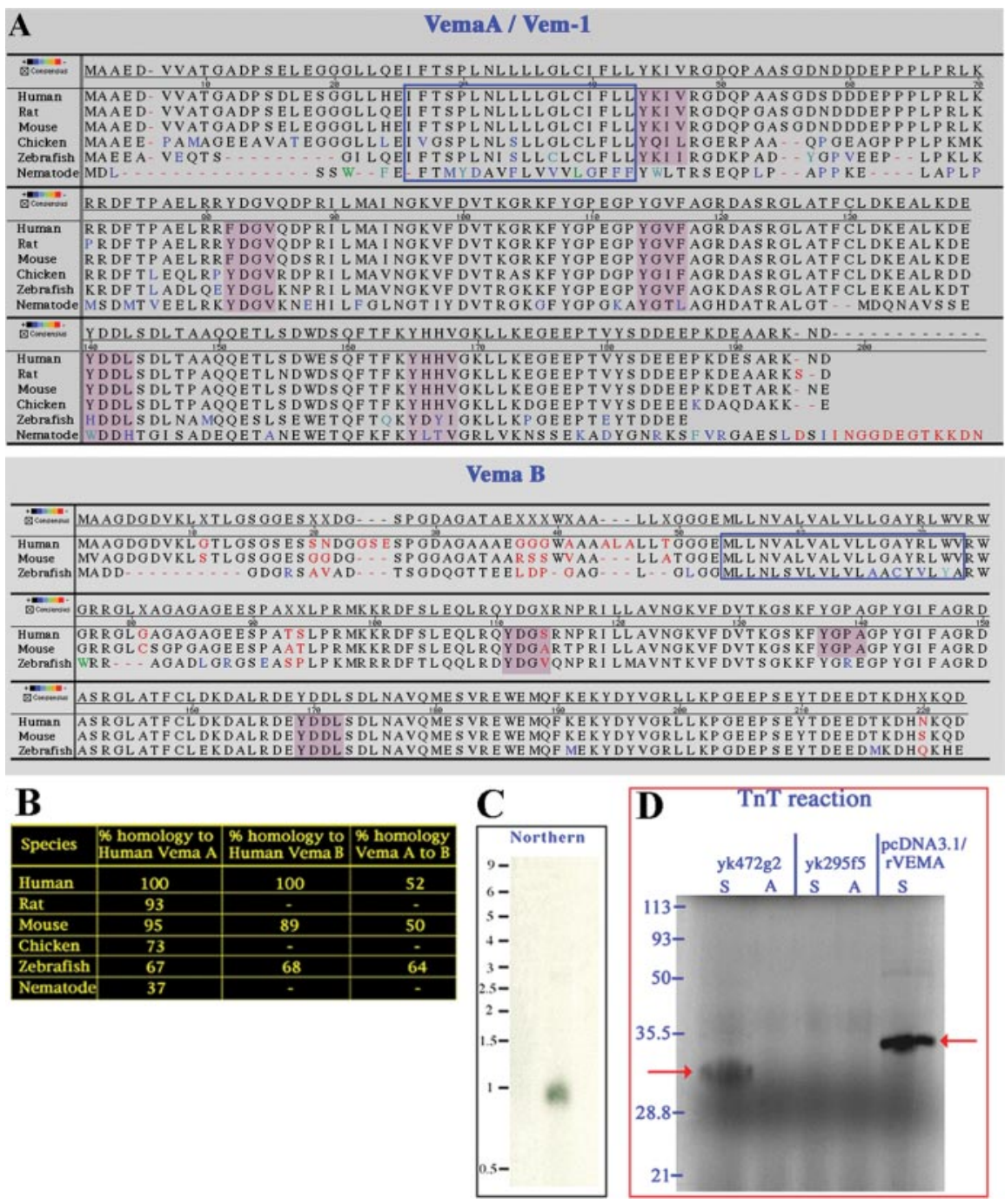

Figure 1. C. elegans VEM-1. A, Amino acid sequence alignments of Vema A/VEM-1 and Vema B homologs. Black letters represent identical amino acids, and red letters represent nonidentical amino acids. Shades of color are used to reflect the degree of similarity-dissimilarity between a given amino acid and its counterpart in the consensus sequence (see scale). The regions boxed by blue lines represent putative transmembrane domains, and the purple shaded regions identify YXX $\phi$ motifs. $B$, Percentiles represent percentage identity as determined by the method of Jotun Hein using the PAM250 residue weight table (Hein, 1990). C, A C. elegans vem- 1 cDNA probe hybridizes to a single $0.8 \mathrm{~kb}$ species in total RNA prepared from mixed-stage larvae. D, In vitro $\mathrm{TnT}$ reaction performed with the C. elegans vem- $1 \mathrm{CDNA}$ clone yk472g2 yields a $32 \mathrm{kDa},{ }^{35} \mathrm{~S}$-labeled protein (left red arrow) that is similar in size to a protein produced from a cDNA encoding the full-length rat Vema A sequence (right red arrow). cDNA clone yk295f5, which contains all of the intronic and exonic sequences present within vem- 1 and, hence, is a probable genomic contaminant that is resistant to transcription-translation, was used as a control for the TnT reaction. S (sense) and A (antisense) indicate the orientation of the CDNA used for transcription.

$5292 \mathrm{~kb}$ fragment (nucleotides 17850-12558) of cosmid C06E1 to a PCR-amplified 826 bp vem-1 fragment from genomic N2 DNA (nucleotides 7780-6954, which encompass exons 1-3; numbering refers to position on cosmid K07E3). The glr-1p::vem-1 construct was injected at $350 \mathrm{ng} / \mu \mathrm{l}$ with the dominant transformation marker pRF4 rol-6(su1006) at $169 \mathrm{ng} / \mu \mathrm{l}$ into vem-1(rz1);rhIs4 or vem-1(rz1);juIs76 animals. The inx-18p::vem-1 construct was generated by ligating a PCR-amplified $1.687 \mathrm{~kb}$ fragment (nucleotides 28787-30474) of cosmid $\mathrm{C} 18 \mathrm{H} 7$ to a PCR-amplified 826 bp vem-1 fragment from genomic N2 DNA (nucleotides 7780-6954, which encompass exons 1-3; numbering refers to position on cosmid K07E3). The inx-18p::vem-1 construct was injected at $331 \mathrm{ng} / \mu \mathrm{l}$ with pRF4 rol-6(su1006) at $169 \mathrm{ng} / \mu \mathrm{l}$ into vem-1(rz1);rhIs4 or vem-1(rz1);juIs76 animals. For above, injection of pRF4 rol-6(su1006) at $169 \mathrm{ng} / \mu \mathrm{l}$ with either $350 \mathrm{ng} / \mu \mathrm{l}$ of the $g l r-1 p$ or $331 \mathrm{ng} / \mu \mathrm{l}$ of the $i n x-18 p$ into vem-1(rz1);rhIs4 or vem-1(rz1);juIs76 animals were used as the controls.
The unc-119::vem-1 construct was generated by ligating a PCR-amplified 826 bp wild-type vem-1 fragment into a $4.478 \mathrm{~kb} \mathrm{BglII} / \mathrm{SacI}$ digested, dephosphorylated pBY103 vector (contains the unc-119 promoter; obtained from $\mathrm{D}$. Pilgrim, University of Alberta, Edmonton, Alberta, Canada). The unc-119p::vem-1 construct was injected at $332 \mathrm{ng} / \mu \mathrm{l}$ with pRF4 rol6(su1006) at $169 \mathrm{ng} / \mu \mathrm{l}$ into oyIs14, rhIs4, and juIs76 animals. Injection of pBY103 at 333 $\mathrm{ng} / \mu \mathrm{l}$ with pRF4 rol-6(su1006) at $169 \mathrm{ng} / \mu \mathrm{linto}$ oyIs14, rhIs4, and juIs76 animals were used as the control. For all of the injections, several extrachromosomal lines were generated and scored.

Statistical analysis. Frequencies of axonal defects between wild-type (or control) nematodes and mutants (or manipulated animals) were compared using a two-sample test of proportions (Stata 6.0 software; Stata, College Station, TX).

In vitro binding analyses. We obtained expression constructs for glutathione $S$-transferase (GST) alone and GST fused to the cytoplasmic domains of UNC-40 [amino acids (aa) 1106-1415] and SAX-3 (aa 898-1273), synthesized a ${ }^{35}$ S-labeled VEM-1 probe, and performed in vitro binding analyses as described previously (Yu et al., 2002). Specifically, molar equivalents of a given GST fusion protein bound to glutathione Sepharose beads were mixed with $19 \mu \mathrm{l}$ of ${ }^{35} \mathrm{~S}$-labeled VEM-1 probe in binding buffer (10 mM Tris, $\mathrm{pH} 7.5,75 \mathrm{~mm}$ $\mathrm{NaCl}, 1 \mathrm{~mm}$ EDTA, $1 \mathrm{~mm}$ DTT, $1 \mathrm{~mm} \mathrm{MgCl}_{2}$, $1 \% \mathrm{NP}-40$, and $10 \% \mathrm{BSA})$. The samples were then incubated end-over-end for 11-14 hr at $4^{\circ} \mathrm{C}$, and the beads were washed four times in the binding buffer (BSA was omitted in the last wash). Bound proteins were separated on a $12 \%$ SDS-PAGE gel that was then fixed, soaked in Amplify (Amersham Biosciences, Piscataway, NJ), dried, and exposed to film for 28-40 hr. These binding experiments were performed three separate times, yielding identical results.

\section{Results \\ Identification of a C. elegans Vema ortholog}

Through extensive database searches, we recently identified vertebrate Vema sequences that can be classified as either Vema A or Vema B (Fig. 1A). The human, mouse, rat, and chicken Vema A sequences correspond to vema cDNAs we identified previously via library screens and the PCR (Runko et al., 1999; Runko and Kaprielian, 2002) (our unpublished results). The human, mouse, and zebrafish Vema B sequences are more highly homologous to each other than to the corresponding Vema A sequences and encode for slightly larger proteins. We recently isolated a partial chick Vema B cDNA (our unpublished results), but rat Vema B has not yet been identified. Inspection of the C. elegans genome sequence identified a $597 \mathrm{bp}$ open reading frame that encodes a putative nematode ortholog of vema $A$ (genetic map position, chromosome $\mathrm{X}$ at -0.62 , matching gene model K07E3.8b), which we named vem-1. Additional analysis by reverse transcription-PCR and 5' RACE indicates that vem-1 has an SL1 trans-splice leader sequence 15 nucleotides upstream of the predicted three-exon 
gene model K07E3.8b. The amino acid sequence of VEM-1 is $37 \%$ identical to the vertebrate Vema A consensus sequence (Fig. $1 B$ ). The Drosophila genome encodes for the following three putative Vema homologs: AAF48534-248 aa, AAF53300192 aa, and AAF46459-287 aa, which are $\sim 48,36$, and $30 \%$ similar, respectively, to a vertebrate Vema A/Vema B consensus sequence (data not shown). However, we cannot conclusively determine whether these predicted fly proteins are likely to be orthologs of Vema A or Vema B. Vema A/VEM-1 and Vema B proteins contain a single transmembrane domain and several conserved tyrosine-based (YXX $\phi$ ) motifs that are thought to mediate the internalization of cell-surface proteins via clathrin-mediated endocytosis (Marks et al., 1997).

We confirmed that C. elegans vem-1 is an expressed gene by sequencing a previously identified cDNA (yk472g2; a generous gift from Yuji Kohara, C. elegans EST Project, National Institute of Genetics, Shizuoka, Japan) and demonstrating that it hybridized to a single $0.8 \mathrm{~kb}$ species in a Northern analysis of total RNA derived from mixed-stage larvae (Fig. 1C). In addition, we subjected yk472g2 to a coupled transcription-translation ( $\mathrm{TnT})$ reaction in vitro and showed that this resulted in the synthesis of a product similar in size to the protein generated from a cDNA representing full-length rat Vema A (Fig. 1D).

\section{A vem-1 reporter gene is expressed in a} small subset of neurons, including AVG, that extend axons into the VNC

To gain insights into the expression pattern of vem-1, we generated a transcriptional reporter transgene in which $\sim 2 \mathrm{~kb}$ of vem-1 sequence, located immediately upstream of the start ATG, was fused to GFP. In transgenic embryos, vem-1::GFP expression was first detected at the beginning of gastrulation in a small number of unidentified cells (data not shown). During later embryonic stages, promoter activity was evident in a subset of putative anterior head neurons (Fig. $2 A-C$ ). Strong GFP labeling was also detectable in what is likely to be the AVG pioneer neuron and in the assembling $\mathrm{VNC}$ as early as the 1.5 -fold stage (Fig. $2 \mathrm{~B}, \mathrm{C}$ ). These findings suggest that vem-1 is expressed by a subset of early developing neurons that extend axons into the right VNC. By the L1 stage, vem-1::GFP activity was clearly detectable in several bilaterally symmetric neurons, including the following: CEPDL/R, RMDVL/R, RIVL/R, AVAL/R, RMDL/R, and RMDDL/R and in the AVG (Fig. 2D). At the L4 stage, vem-1::GFP continues to be expressed by a subpopulation of nerve ring neurons and by AVG (Fig. 2E). The presence of GFP along the dorsalmost axon of the VNC is consistent with the AVG neuron expressing vem-1::GFP (Fig. 2 E). Similarly, the GFP labeling observed in a small subset of more ventrally positioned right VNC-associated axons identifies AV (anterior ventral) interneurons as additional sites of
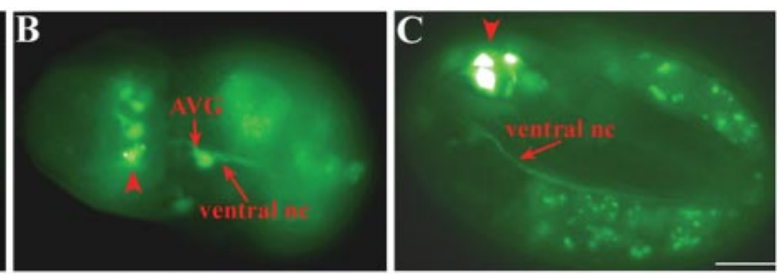

Figure 2. A vem-1 reporter gene is expressed in a small subset of neurons that extend longitudinally projecting axons into the right VNC in C. elegans embryos and larvae. $A-C$, vem-1::GFP is expressed in embryonic neurons before the comma stage $(A)$, at 1.5-fold stage $(B)$, and at the threefold stage ( $C$. Red arrowheads point to vem-1::GFP-expressing head neurons. Expression ons that compose more ventral regions of the VNC The animal depicted in this micrograph was selected because it provided a particularly good example of GFP labeling along the extent of AVG- and VNC-associated axons. However, this was one of the 列 -1 protein fused to GFP driven by the vem-1 promoter, revealed VEM-1::GFP expression in a subset of nerve ring neurons that extend axons into the VNC (blue arrow), as well as the AVG (blue arrow). Note that the non-neuronal labeling that was variably observed in the transcriptional reporter line was mostly absent in the VEM-1::GFP translational line. Scale bars, $10 \mu \mathrm{m}$.

vem-1::GFP transgene expression (Fig. 2E). Consistent with vem-1::GFP expression being restricted to a subset of longitudinally projecting interneurons, axons extending from circumferentially projecting motor neurons were not labeled (Fig. 2). Notably, the labeling of the intestine and pharyngeal muscles (Fig. $2 E$ ) was highly mosaic, because it was only detected in a small subset of transgenic animals. In contrast, labeled neurons were observed in each transgenic animal harboring the vem-1::GFP transcriptional reporter construct. Therefore, we consider the non-neuronal labeling to be nonspecific and provisionally attribute it to background activity associated with the plasmid used to generate this reporter construct (ftp://www.ciwemb.edu/ pub/FireLabInfo/FireLabVectors/1999_Vector_Kit/Vec99_Docs/ Vec99Doc.RTF).

The vem-1 expression pattern described above is based on the use of a transcriptional reporter construct, an approach that has been used previously to accurately document the distribution of a variety of neuronally expressed genes (Tsalik et al., 2003). However, because the transgene we used does not include the entire 


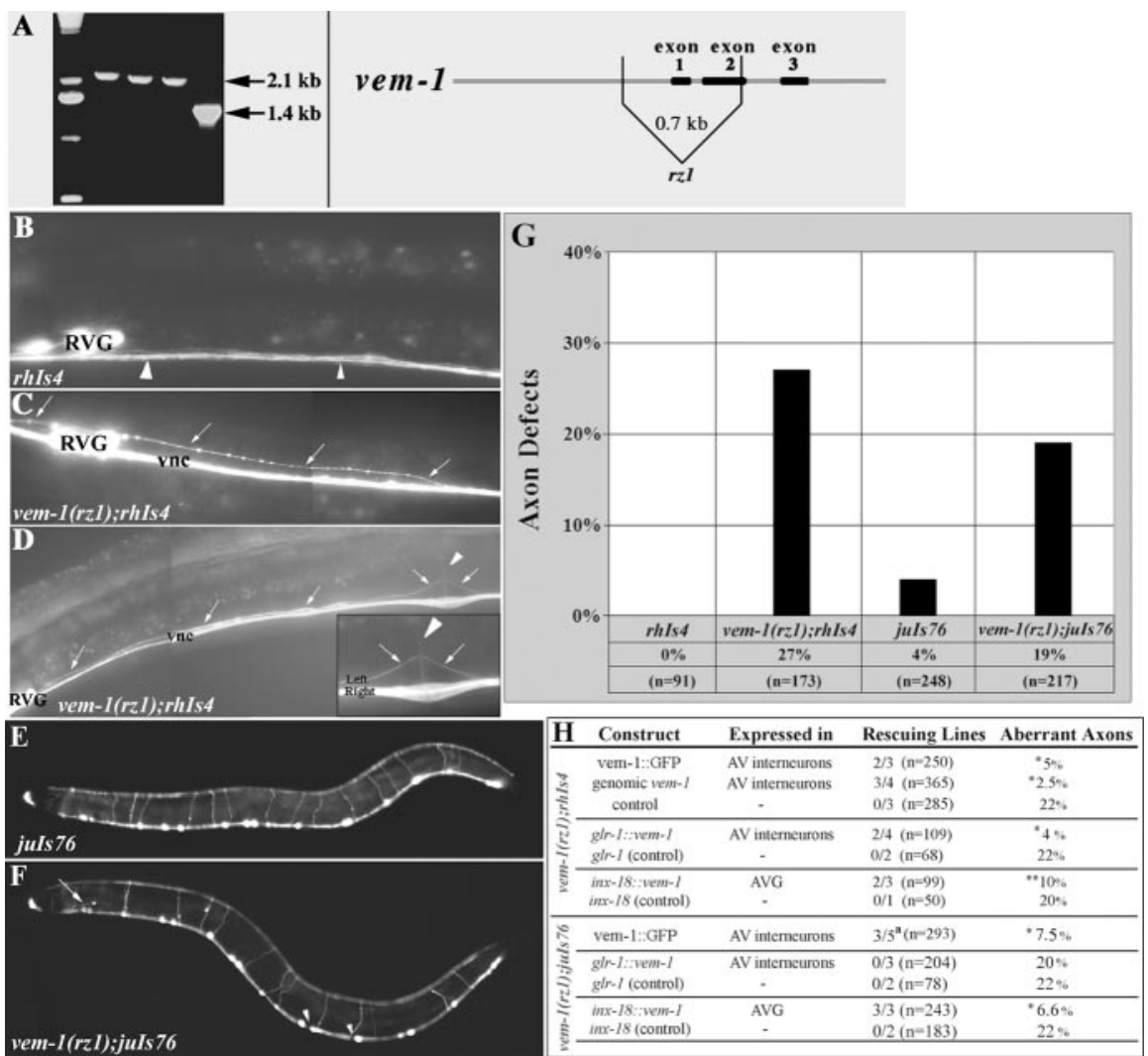

Figure 3. vem-1(rz1) mutants display clear defects in the patterning of a subset of VNC-associated interneuron axons and motor neuron axons. $A$, vem-1 mutant isolated through deletion library screening. Each of the lanes on this gel (except the extreme left-hand lane, molecular weight markers) contains the product obtained from performing a two-step PCR using nested vem-1 primers (see Materials and Methods) and DNA derived from an individual 96-well plate of mutagenized worms. Although the $2.1 \mathrm{~kb}$ products represent intact vem-1, DNA sequencing indicates that the $1.4 \mathrm{~kb}$ product represents a truncated form of vem-1, which lacks exon 1 and essentially all of exon 2. Schematic view of vem-1 structure and the location of the $0.66 \mathrm{~kb} r z 1$ mutant-associated deletion. $B$, In wild-type animals, the $r h / s 4$ reporter labels anteriorly located neurons that extend posteriorly projecting axons into the right tract of the VNC to form a thick axon-rich fascicle (arrowheads). C, In some vem-1(rz1) mutants, an axon, or a thin fascicle of axons emanating from the nerve ring, failed to extend ventrally and enter the right tract of the VNC. Instead, this axon(s) extended posteriorly along the longitudinal axis in an inappropriate lateral position immediately dorsal to the RVG (arrows). D, Semiventral view. In other vem-1(rz1) mutants, mispositioned axons (arrows) aberrantly traveled in the left (as opposed to the right) tract of the VNC. $E$, In wild-type animals, the juls76 reporter labels DD and VD motor neurons that initially extend longitudinally along the VNC and then project circumferentially and join the longitudinally oriented DNC. F, In some vem-1(rz1) mutants, an axon (arrow) that initially projected dorsally prematurely turned into the longitudinal plane and failed to join the DNC. Axonal gaps (arrowheads) in the VNC were observed within the same animal. G, Quantification of axonal defects (lateral axons and aberrant placement of axons in the left VNC, combined) observed in vem-1(rz1);rhls4 animals $(p<0.001)$ and axonal defects (axonal gaps and misprojected circumferential axons in the lateral domain or left side, combined) observed in vem-1(rz1);juls76 animals $(p<0.001)$. $H$, Rescue of aberrant axon phenotype using a variety of expression constructs. Constructs were injected into vem-1(rz1);rh/s4 or vem-1(rz1);juls76 animals as indicated. * indicates the percentage calculated from the rescued lines; ${ }^{* *}$ indicates the percentage calculated from the partially rescued lines. ${ }^{a}$ indicates that one line showed partial rescue $(12.5 \%)$ of the defects. $p<0.001$ for all.

vem-1 gene, it may not fully reflect transcriptional and posttranscriptional controls on vem-1. To confirm the observed expression pattern, we generated a VEM-1::GFP translational reporter construct by inserting GFP in frame before the stop codon in the VEM-1 protein sequence. Transgenic animals expressing either the translational or the transcriptional vem-1 reporter displayed the same labeling pattern. Importantly, animals harboring the translational reporter confirm that VEM-1 is expressed by a subset of nerve ring neurons and the AVG pioneer neurons, each of which extend GFP-labeled axons into the VNC (Fig. $2 F$ ).

\section{Isolation of the vem-1(rz1) mutant}

A single putative vem- 1 null mutant, lacking $\sim 0.66 \mathrm{~kb}$ of the vem-1 locus (eliminating exon 1 and most of exon 2; see Materials and Methods) and designated vem-1(rz1), was isolated by screening pools of mutagenized worms for deletions in the vem-1 gene using a nested PCR strategy (Fig. 3A). vem-1(rz1) was phenotypically wild type, displaying no observable defects in the following: body size or shape, morphology, response to touch, embryonic viability, feeding, mating, or locomotion. In addition, no obvious defects in the gross positioning of several classes of neurons were observed when vem-1(rz1) mutants were examined in a variety of GFP reporter backgrounds (see below).

\section{vem-1(rz1) phenotype: VNC-associated interneuron axon patterning defects} Given that vem- 1 is expressed in a subset of nerve ring-associated neurons that extend posteriorly projecting axons along the right VNC (Fig. 2F), we investigated the possibility that the patterning of these axons might be disrupted in vem-1(rz1) animals. Specifically, we crossed vem-1(rz1) animals with wild-type worms expressing the $g l r-1:: G F P$ transgene (strain rhIs4), which labels 14 interneurons: AVAL/R, AVBL/R, AVDL/R, AVEL/R, AVJL/R, DVC, PVCL/R, as well as AVG, that each extend longitudinally projecting axons along the right VNC (Hart et al., 1995; Maricq et al., 1995). In lateral views of wild-type $g l r-1:: G F P$-expressing animals, a single thick axon fascicle is visible within the right VNC (Figs. 3B, 4A). In contrast, two types of axonal patterning defects were observed in a subset of vem-1(rz1);glr-1::GFP mutant animals. First, one or more posteriorly projecting axons were located in aberrant lateral positions rather than within the VNC (Figs. 3C, 4A). This observation suggests that vem-1 is required for the guidance of $g l r-1:: G F P$-expressing axons to the ventral midline. However, vem-1 must not generally be required for this type of guidance event because the ventrally directed pathfinding of axons emanating from a variety of other neurons (e.g., AVM) (Hamelin et al., 1992; Chan et al., 1996; Yu et al., 2002) is not perturbed in vem-1 mutants (Fig. $4 C)$. Second, in ventral views of some vem-1(rz1) mutant animals, $g l r-1:: G F P$-labeled axons were not appropriately restricted to the right $\mathrm{VNC}$ but, rather, were located on both the right and left sides of the midline (Figs. 3D, 4A). This particular phenotype, which was most apparent in the immediate vicinity of the vulva, in which the left and right sides of the $\mathrm{VNC}$ are well separated (Fig. 3D, inset), supports a role for vem-1 in segregating glr-1::GFP-labeled axons to the right tract of the VNC. Collectively, mispositioned lateral axons and inappropriate axon crossover events were observed in $27 \%$ of $v e m-1(r z 1)$ mutant animals (Fig. 3G). A genomic fragment representing the entire vem-1 locus rescued the vem-1 mutant-associated, glr-1::GFPexpressing axon patterning defects in three of four transformed 
lines (Fig. 3H). Importantly, the VEM-1::GFP translational reporter (fulllength VEM-1 protein fused to GFP driven by vem-1 promoter) also rescued glr-1::GFP-expressing axon patterning defects in two of three transformed lines (Fig. $3 H$ ). Because vem- 1 is expressed in AVG, we also asked whether AVG axon pathfinding is perturbed in vem-1 mutant animals. Specifically, we crossed vem-1(rz1) mutants with animals expressing either the vem-1::GFP transcriptional reporter, which is a robust marker of AVG (Fig. 2), or the inx-18::GFP reporter that selectively labels AVG and its posteriorly projecting pioneer axon (Fig. 4C) (Bulow et al., 2004). In both cases, AVG axon pathfinding was unaffected by the vem-1 mutation (Fig. 4C) (data not shown).

vem-1 is expressed in a subset of VNCassociated interneurons, including AVG (Fig. 2). Therefore, we performed cell type-specific rescue experiments to determine whether vem-1 activity is required in these cells for the proper pathfinding of VNC-associated axons. The expression of vem-1 under the control of the glr-1 promoter (drives expression in the command interneurons and AVG) rescued the lateral axon phenotype in two of four lines (Fig. $3 H$ ). To potentially distinguish between requirements for vem-1 activity in the command interneurons versus AVG, we subsequently asked whether vem-1 under the control of the AVG-specific inx-18 promoter would also rescue aberrant pathfinding. This resulted in a partial rescue (two of three lines) of the lateral axon phenotype (Fig. $3 H$ ). Together, these observations suggest that vem-1 may operate both cell autonomously in interneurons and cell nonautonomously through AVG, to regulate the guidance of posteriorly projecting VNC-associated axons. The latter possibility is supported by the observation that the AVG axon pioneers the right VNC and presumably provides follower axons with a key source of guidance information (Durbin, 1987; Wightman et al., 1997; Hutter, 2003). It is important to note that these cell autonomy-nonautonomy arguments are only valid if vem-1 is expressed by $g l r-1:: G F P$-expressing interneurons and AVG, respectively. Indeed, a VEM-1::GFP fusion protein is localized to a subset of VNC-associated interneurons, including AVG (Fig. $2 F$ ).

vem-1(rz1) phenotype: VNC-associated motor neuron axon patterning defects The 19 GABAergic DD and VD motor neurons are key components of a circuit that mediates backward and forward
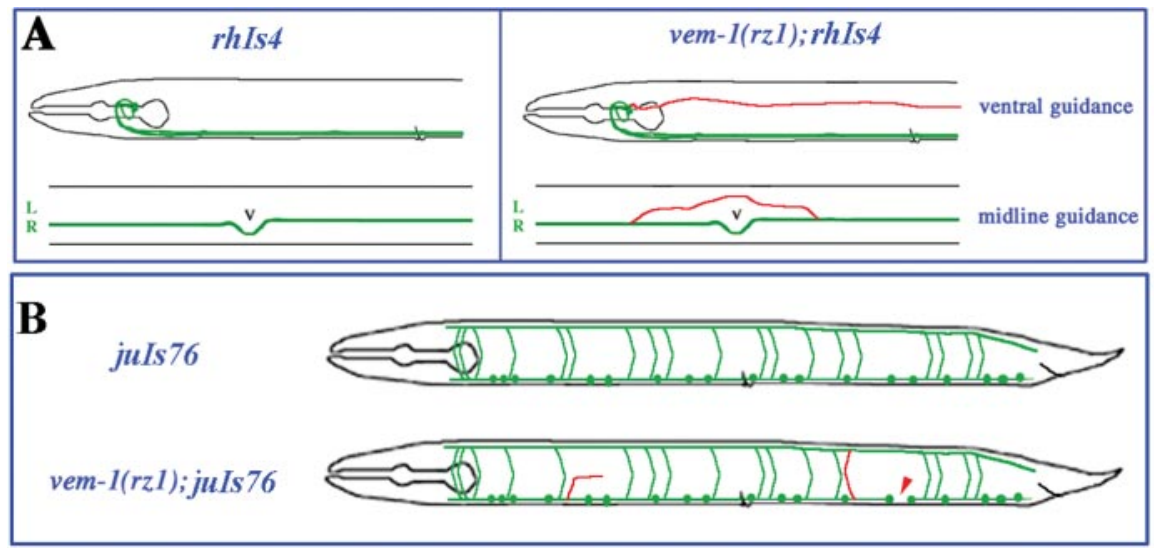

\section{CLASSES OF NEURONS UNAFFECTED IN vem-1(rz 1$)$ MUTANTS}

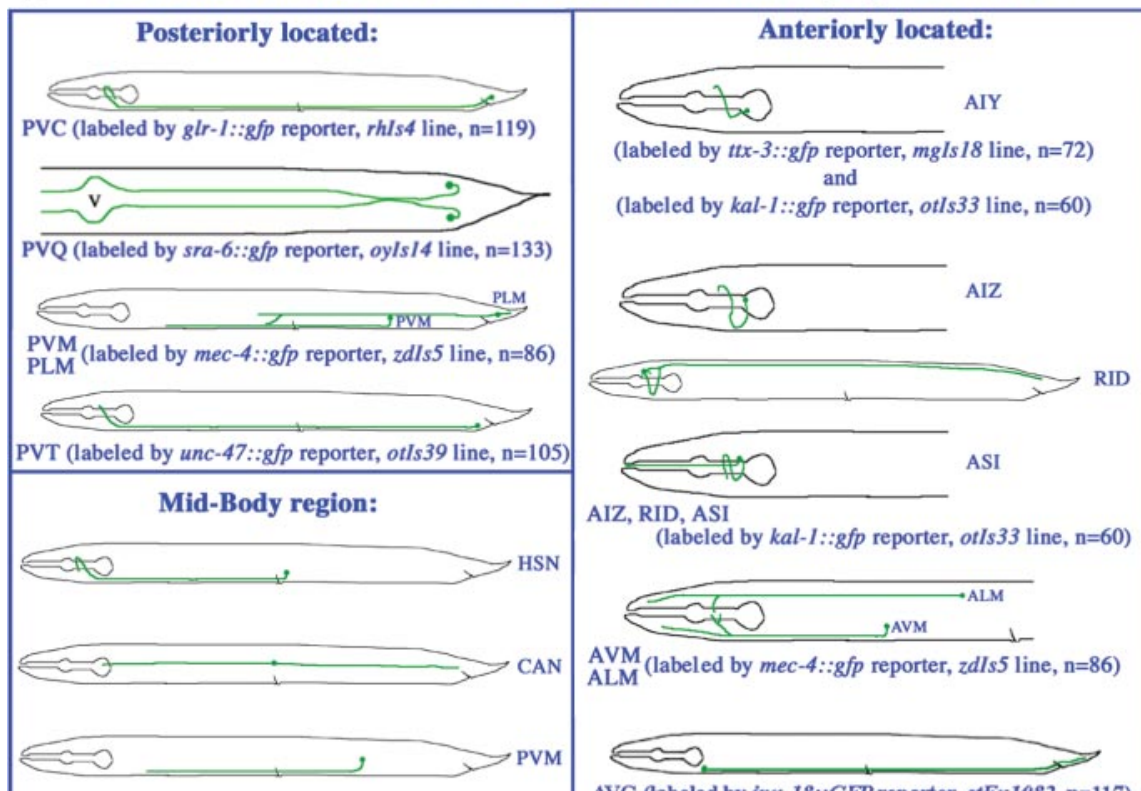

HSN, CAN, PVM

(labeled with kal-1::gfp reporter, otls33 line, $\mathrm{n}=60$ )

Motor Neurons:
$\mathrm{20}_{\mathrm{NC}} \mathrm{vC}$

(labeled by lin-11::gfp reporter, $n I s 106$ line, $\mathrm{n}=40$ )

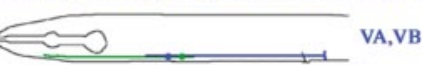

(labeled by unc-4::gfp,wdIs3 line, $\mathrm{n}=92$ )
AVG (labeled by inx-18:: GFP reporter, otEx 1082, $\mathrm{n}=117$ )

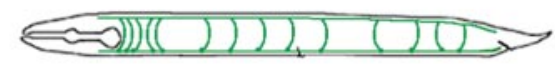

DA, DB (labeled by unc-129::GFP, evIs82b line, $\mathrm{n}=87$ )
Figure 4. Phenotype of specific neurons-axons in vem-1(rz1) mutants. $A$, vem-1 is required for the proper guidance of longitudinally projecting VNC-associated axons. In the vem- 1 mutant, a subset of posteriorly projecting axons fails to properly extend toward the VNC (ventral guidance) or inappropriately crosses the midline within the VNC (midline guidance). Each of the schematics represents a wild-type or vem- 1 mutant animal in the rh/s 4 reporter background. A lateral view of the anterior half of the animal is shown at the top, whereas a ventral view at the midbody region is shown at the bottom. Mispositioned lateral axons (top) or axons that aberrantly crossed the midline (bottom) are indicated in red. L, Left tract of the VNC; R, right tract of the VNC; $V$, vulva. $B$, vem- 1 is required for the proper guidance of $D$-type motor neuron axons. In the vem- 1 mutant, axon defects included gaps in longitudinal axon tracts (red arrowhead) and either abnormally oriented commissures resulting from lateral wandering of circumferentially projecting axons or the inappropriate extension of these axons along the left side of the animal (both are indicated as red axons). C, Classes of neurons-axons whose development are unaffected in vem-1(rz1) mutants. All of the worm schematics, except for the one depicting the trajectory of PVQ-associated axons (top left; sra-6::GFP reporter; ventral view), are shown in the lateral view. Axons are green. Each of the identified neurons exists as left-right pairs, except for PVT, PVM, RID, and the various motor neurons. There are $12 \mathrm{VA}, 11 \mathrm{VB}, 6 \mathrm{VC}, 7 \mathrm{DA}$, and $7 \mathrm{DB}$ motor neurons. In the schematic depicting the expression of the lin-11::GFPreporter, only one VC motor neuron (green) is shown. In the unc-4::GFPreporter schematic, single VA (green) and VB (blue) motor neurons are shown. In each schematic, $n$ denotes the number of vem-1(rz1) mutants examined in a given GFP reporter background $(p<0.001)$. 
movements and are labeled by the unc-25::GFP reporter (strain juIs76) (Figs. 3E, 4B) (Jin et al., 1999). In wild-type animals, each motor neuron projects an anteriorly directed axon along the VNC that bifurcates and gives rise to a longitudinal projection that continues to grow along the VNC, as well as a circumferentially directed commissural projection that extends toward the DNC (White et al., 1986). Seventeen of the 19 neurons project commissures along the right side of the body, whereas the DD1 and VD2 motor axons project along the left side. After reaching the dorsal midline, all of these axons bifurcate again to extend both anteriorly and posteriorly directed projections along the DNC (White et al., 1986). vem-1(rz1) mutants examined in the unc-25::GFP background displayed a variety of DD/VD motor axon defects, including the following: axonal gaps resulting from defects in axonal extension along the VNC (Figs. 3F, $4 B$ ) or DNC (data not shown), incomplete or abnormal circumferential projections (Figs. $3 F, 4 B$ ), and inappropriate circumferential extensions along the left side of the body (data not shown). Collectively, these defects were observed in 19\% of vem-1(rz1) mutant animals (Fig. $3 G$ ). Importantly, the expression of a VEM-1::GFP fusion protein under the control of its own promoter (translational reporter) rescues these motor axon pathfinding defects (Fig. $3 H$ ). Given that vem-1 does not appear to be expressed in motor neurons, one interpretation of these findings is that AVGassociated vem-1 cell nonautonomously regulates the pathfinding of motor neuron axons. Our finding that the selective expression of vem-1 in AVG rescues motor axon pathfinding defects (Fig. $3 H$ ) is consistent with this notion. Furthermore, the AVG neuron represents a key source of guidance information for pathfinding D-type motor neurons (Durbin, 1987; Huang et al., 2003; Hutter, 2003).

\section{vem-1(rz1) phenotype: unaffected neurons-axons}

To test the possibility that vem-1 is required for the proper development of axons other than the restricted subset of $g l r-1:: G F P$ expressing interneuron axons and unc-25::GFP-labeled motor neuron axons, we crossed the vem-1(rz1) mutant with wild-type worms expressing GFP in other classes of neurons/axons (Fig. 4). Initially, we examined vem-1(rz1) animals in the pan-neuronal/ axonal unc-119::GFP reporter background (strain edIs20) (Maduro and Pilgrim, 1995; Wightman et al., 1997; Knobel et al., 2001). Despite extensive GFP labeling in most longitudinally and circumferentially projecting axons, the lateral axon and axon crossover defects described above were restricted to a subset of posteriorly projecting axons emanating from nerve ringassociated neurons (data not shown), just as we observed in the glr-1::GFP background. Consistent with the anteriorly projecting axon-specific defects described above, additional analyses revealed that PVC-, PVQ-, PLM-, and PVT-associated axons, which each extend from posteriorly located neurons, traveled along wild-type trajectories in a vem-1 mutant background (Fig. 4). Using a variety of other GFP reporter lines, we demonstrated that the following classes of neurons/axons develop normally in vem-1 (rz1) mutant animals: midbody neurons HSN, CAN, PVM, ALM, and AVM, which extend anteriorly or posteriorly projecting axons; anteriorly located neurons AIY, AIZ, ASI, and RID, which extend axons into the nerve ring or the DNC; ventrally located motor neurons VA, VB, and VC, which extend longitudinally projecting axons along the VNC; and ventrally located motor neurons DA and DB, which extend circumferential axons dorsally to the DNC or longitudinal axons along the VNC (Fig. 4). In addition, the anteriorly located AVK neurons that extend posteriorly projecting axons along the right and left VNC (strain $b w I s 2 ; f l p-1:: G F P$ reporter) were unaffected in a vem-1 mutant background (data not shown).

\section{Disruption of vem-1 function by conventional or hairpin RNAi phenocopies vem-1(rz1) mutants}

To independently confirm that vem-1 is required for the proper assembly of the VNC, we used both conventional and induciblehairpin RNAi (Fire et al., 1998; Montgomery and Fire, 1998; Montgomery et al., 1998; Hunter, 2000) to interfere with vem-1 function. In the conventional approach, we generated dsRNA corresponding to exons 1-2 of vem-1, as well as the appropriate intervening intronic sequences (see Materials and Methods), and examined the consequences of vem-1 dsRNA injections in the glr-1::GFP reporter line. Consistent with our phenotypic analysis of the vem-1 mutant, $23 \%$ of the vem-1 dsRNA-injected animals exhibited mispositioned lateral axons, inappropriate midline crossing events, and a variety of motor neuron axon pathfinding errors (data not shown). To perform the hairpin vem-1 RNAi experiments, we made use of a recently developed strategy aimed at efficiently introducing inducible or hairpin dsRNA into the $C$. elegans nervous system (Tavernarakis et al., 2000). Using this approach, we observed vem-1 mutant- and vem-1 dsRNA-like guidance defects at very similar frequencies (data not shown).

\section{Misexpression-overexpression of vem-1 leads to axonal defects that resemble those exhibited by vem-1(rz1) mutants}

To assess the possible effects of increased levels/ectopic expression of vem-1 on VNC development, we placed vem-1 under the control of the $u n c-119$ promoter (see Materials and Methods) to drive expression in all of the neurons and examined the transgenic animals in a variety of reporter backgrounds. In the rhIs4 ( $g l r-1:: G F P)$ or juIs76 (unc-25::GFP) reporter backgrounds, panneuronal expression of vem-1 resulted in axonal patterning defects that resembled, and were just as penetrant as, those observed in the vem-1(rz1) mutant (Fig. 5). Specifically, 23\% of vem-1(+); juIs76 animals (Fig. $5 \mathrm{H}$ ) displayed aberrantly positioned lateral axons (Fig. 5B), misprojected left-sided commissures (Fig. 5C), and/or axonal gaps in the VNC (Fig. $5 D$ ) or DNC (Fig. 5E), whereas $21 \%$ of vem-1(+);rhIs4 animals exhibited the aberrant lateral axon phenotype (Fig. $5 H$ ) (data not shown) or the inappropriate crossover of axons from the right into the left tract of the VNC (Fig. 5G). In the sra-6::GFP reporter background (strain oyIs14) (Troemel et al., 1995), inappropriate midline crossing events and aberrantly positioned lateral PVQ neuron-derived axons were observed in $14 \%$ of the animals examined (Fig. $5 \mathrm{H}$ ) (data not shown). Notably, vem-1(rzl) mutant animals did not exhibit defects in PVQ axon pathfinding (Fig. 4C). Together, these findings suggest that inappropriately widespread expression of vem-1 disrupts the pathfinding of particular classes of longitudinally and circumferentially projecting axons, including some that are not affected in vem-1 mutant animals.

\section{vem-1 is likely to act in the $u n c-40$ pathway to regulate the dorsoventral guidance of interneuron axons, as well as D-type motor neuron axon pathfinding}

In vem-1 mutants, some glr-1::GFP-expressing axons either fail to properly extend to the VNC or inappropriately join the left tract of the VNC (Fig. 4A). These defects suggest roles for vem-1 in the long-range attraction of axons to the ventral midline and in the segregation of axons to the appropriate side of the VNC. Previous studies have shown that UNC-40 functions as an attractive UNC-6 receptor (Chisholm and Tessier-Lavigne, 1999; Wadsworth, 2002), that sax-3 mutants exhibit aberrant midline cross- 
over events (Zallen et al., 1998, 1999), and that unc-40 (Chan et al., 1996) and sax-3 (Zallen et al., 1998) are expressed by at least a subset of VNC-associated interneurons. As a first step toward determining whether vem-1 might genetically interact with $u n c-40$ and/or sax-3, we examined the phenotype of $u n c-40(e 271)$ and sax3 (ky123) null mutants in the glr-1::GFP background. In both unc-40(e271);rhIs4 (Fig. 6C) and sax-3(ky123);rhIs4 (Fig. 6E) animals, a subset of posteriorly projecting axons assumed aberrant lateral positions. Anteriorly projecting PVC axons were also observed in inappropriate lateral positions in $u n c-40$ (e271) (Fig. 6D), but not in sax$3(\mathrm{ky} 123)$ (Fig. $6 E$ ) or in vem-1(rz1) (Fig. $6 B)$, mutant backgrounds. In addition to the lateral axon phenotype, axons were inappropriately positioned on both the right and left sides of the VNC in a small percentage of sax-3, but not unc-40, null mutant animals (data not shown). It has been shown previously that $\sim 40 \%$ of unc- 40 null mutants displayed defects in the pathfinding of D-type motor axons that included wandering along the DV axis and the inappropriate projection of commissures on the left side of the body, as well as prematurely terminated longitudinally oriented growth that results in gaps in the VNC and DNC (Huang et al., 2002). In this study, we have shown that $19 \%$ of vem-1 null mutants exhibited similar motor neuron-axon defects in the juIs76 reporter background (Fig. $3 G$ ).

To test the possibility that vem-1 genetically interacts with $u n c-40$ and/or sax-3 to regulate interneuron axon pathfinding, we constructed double mutants using either unc-40 or sax-3 null alleles and the vem-1 null mutant, and we scored for the aberrant lateral axon phenotype in the glr-1::GFP background. The following genetic criteria were used to interpret the double-mutant analyses: (1) if two genes act exclusively in the same pathway, the double-mutant phenotype should be similar to that of the strongest single null mutant, and (2) if two genes act in parallel pathways, additive or synergistic effects may be observed. These analyses revealed that vem-1 and unc- 40 are likely to act in the same genetic pathway for the guidance of interneuron axons to the VNC because the unc-40(e271);vem-1(rz1) and unc-40(e1430);vem-1 (rz1) mutants were as defective as either single null mutant (Fig. $6 H$ ). However, it appears that vem-1 and sax-3 act in parallel pathways for guiding axons to the VNC because additive effects were observed in the sax-3(ky123);vem1(rz1) double mutants (Fig. $6 \mathrm{H}$ ). Our data also suggest that vem-1 and $u n c-40$ operate in the same genetic pathway for the guidance of D-type motor neuron-axons because unc-40(e271); vem-1(rz1) or unc-40(e1430);vem-1(rz1) mutants examined in the juIs76 background were as defective as the strongest single null mutant, unc-40 (Fig. 6I).
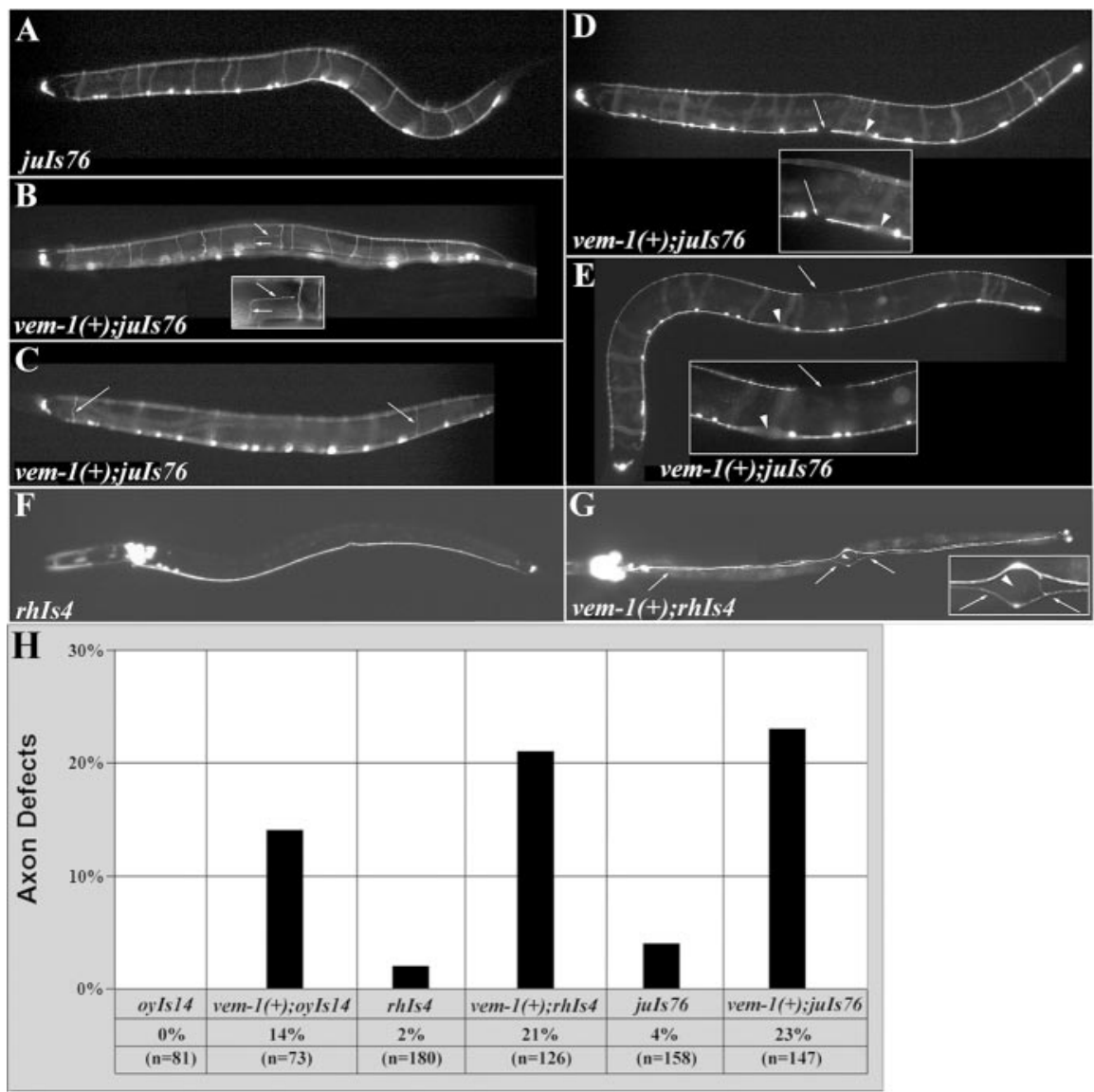

Figure 5. Overexpression of vem- 1 leads to axon pathfinding defects. $A$, In control juls76 animals (uninjected or pBY103 injected), DD and VD motor neurons initially extend longitudinally oriented axons along the VNC and then project circumferendemarcate an axon that turned prematurely into the longitudinal plane and failed to join the DNC. Inset in $B$ is a higher magnification of the aberrant axon. C, The left side of the worm is in focus. Most of the motor neurons project circumferentially on the right to an aberrant motor axon located near the posterior end of the animal that projected circumferentially on the left, as opposed to int, side. $D$, The arrow points to an axonal gap in the VNC. $E$, The arrow points to an axonal gap in the DNC. The inset is control rh/s4 animals (uninjected or pBY103 injected), interneurons extend posteriorly projecting axons into the right tract of the left tract of the VNC. $G$, Inset, A higher magnification of the aberrant axon in the vulva region. $H$, Summary of axonal defects observed in vem-1(+);oyls 14, vem-1(+);rhls4, and vem-1(+);juls76 animals $(p<0.001)$.

\section{VEM-1 binds UNC-40}

It has been demonstrated recently that the cytoplasmic domains of UNC-40 and SAX-3 are capable of associating in vitro (Yu et al., 2002). Previously, the Xenopus turning assay was used to show that direct interactions between the cytoplasmic domains of vertebrate DCC and Robo mediate the silencing of netrin attraction in vitro (Stein and Tessier-Lavigne, 2001). Together, these findings suggest that the observed genetic interactions between UNC-40 and SAX-3 may be mediated by direct physical interactions between these two guidance receptors (Yu et al., 2002). By analogy, our finding that vem-1 is likely to operate in the unc-40, but not the sax-3, pathway suggests that VEM-1 might physically interact with UNC-40, but not SAX-3, in some cellular settings. To test these possibilities, we asked whether ${ }^{35} \mathrm{~S}$-labeled in vitro translated VEM-1 is capable of binding GST fusion proteins representing the cytoplasmic domains of UNC-40 or SAX-3. Consistent with our genetic data, we found that VEM-1 associates with the cytoplasmic domain of UNC-40 but not SAX-3 (Fig. 7). 


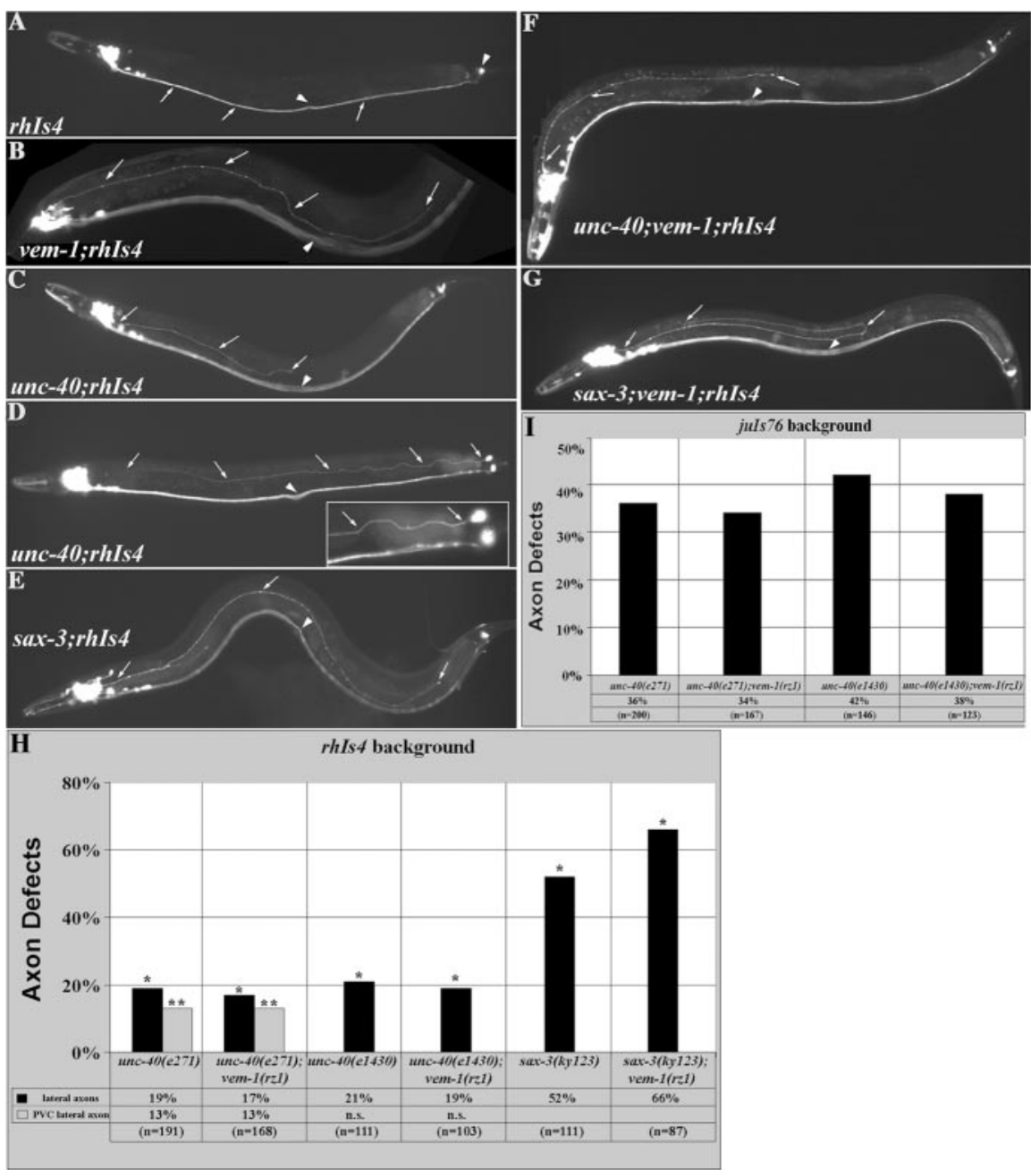

Figure 6. unc-40 and sax-3 mutants exhibit vem-1(rz1)-like axon patterning defects. In wild-type animals, the rh/s4 reporter labels VNC-associated interneurons-axons (arrows), as well as the bilaterally symmetric PVC tail neurons (right arrowhead) that extend anteriorly projecting axons along the right VNC. $B, C$, In some vem-1(rz1);rh/s4 and unc-40(e271);rh/s4 animals, a subset of axons from the nerve ring fail to project ventrally into the VNC and instead extend posteriorly in an inappropriate lateral position (arrows). D, In some unc-40(e271);rh/s4 animals, the PVCL axon also fails to properly extend to, and join, the VNC and instead travels in an aberrant lateral position (arrows) to the nerve ring. Inset, The initial segment of the trajectory followed by the mispositioned PVCL axon (arrows) at higher magnification. E, In 52\% of the sax-3(ky123);rh/s4 animals examined, a small subset of posteriorly projecting axons that arise from nerve ring-associated neurons extend in an aberrant lateral position toward the tail (arrows). F, unc-40(e271);vem-1(rz1);rh/s4 animals are indistinguishable from unc-40(e271);rh/s4 animals for the aberrant lateral axon phenotype. Arrows point to an axon that fails to turn ventrally to join the VNC and instead travels longitudinally in an inappropriate lateral position and terminates just before reaching the vulva. G, sax-3(ky123);vem-1(rz1);rh/s4 animals exhibit an aberrant lateral axon phenotype that is much more penetrant than that observed in either the vem-1 or sax-3 null mutants alone. Arrows point to an aberrant lateral axon that turns dorsally after reaching the vulva to project anteriorly toward the head. $A-G$, The midbody arrowhead marks the position of the vulva. $H$, Quantification of axonal defects observed in unc-40(e271);rh/s4, unc40(e271);vem-1(rz1);rhls4, unc-40(e1430);rhls4, unc-40(e1430);vem-1(rz1);rhls4, sax-3(ky123);rhls4, and sax-3(ky123);vem1(rz1);rh/s4 animals $\left({ }^{*} p<0.001 ;{ }^{* *} p<0.01\right)$. n.s. indicates that the PVC lateral axon defect was not scored in unc-40(e1430); rh/s4 and unc-40(e1430);vem-1(rz1);rh/s4 animals. I, Quantification of axonal defects observed in unc-40(e271);juls76, unc40(e271);vem-1(rz1);juls76, unc-40(e1430);juls76, and unc-40(e1430);vem-1(rz1);juls76 animals ( $p<0.001)$.

\section{Discussion}

In both vertebrates and invertebrates, the CNS midline is a key intermediate target for pathfinding axons. Midline-associated cells and axons represent key sources of both attractive and repellent cues that guide axons to, and segregate them to either side of, the CNS (Kaprielian et al., 2001). The evolutionarily conserved UNC-40/DCC and SAX-3/Robo cell-surface receptors mediate the actions of midline-associated chemoattractants and chemorepellents, respectively. In this report, we identify vem-1 to be the C. elegans ortholog of vertebrate Vema A, a novel membrane- associated protein expressed at midline choice points (floor plate and optic chiasm) in the developing rodent CNS (Zhu et al., 1998; Runko et al., 1999; Runko and Kaprielian, 2002). We show that vem-1 is expressed by the AVG midline pioneer neuron, as well as by a subset of nerve ringassociated neurons, each of which project axons into the right tract of the VNC. In addition, the phenotype of a vem-1(rz1) mutant reveals that VEM-1 is required for the proper guidance of VNC-associated interneuron and motor neuron axons. The results of double mutant and in vitro binding analyses further suggest that vem-1 functions in the $u n c-40$ pathway.

\section{vem-1 is required for interneuron and motor neuron axon guidance}

In vem-1 mutants, some posteriorly projecting $g l r-1:: G F P$-expressing interneuron axons frequently fail to extend to the ventral midline. Instead, they project away from the nerve ring at inappropriate lateral positions. vem-1 is likely to be expressed by a subset of these neurons, and expressing vem-1 under the control of the glr-1 promoter (or expressing a VEM-1::GFP fusion protein under the control of the vem-1 promoter) in vem-1(rz1) mutants fully rescued the lateral axon phenotype. These results suggest that vem-1 has a cellautonomous role for VNC-associated interneuron axon guidance. However, the finding that the lateral axon phenotype is partially rescued by selectively directing the expression of vem-1 to AVG in a vem-1 mutant background suggests a cell-nonautonomous role for vem-1. Because increased levels of vem-1 in neurons lead to axon patterning defects (Fig. 5), the partial rescue in the latter experiment likely results from the presumed higher levels of vem-1 expressed by the AVG-specific promoter, inx-18, compared with the glr-1 promoter. A nonautonomous role for vem-1 is further supported by our finding that, although vem-1 is likely to be expressed by AVG, AVG axon pathfinding is normal in vem-1 mutants, and animals that lack AVG display VNC-associated axonal patterning defects (Durbin, 1987; Hutter, 2003). Thus, in addition to presumably acting cell autonomously in a subset of glr-1::GFP-expressing neurons, VEM-1 might also function cell nonautonomously in the AVG pioneer neuron to control the guidance of VNC-associated follower axons, possibly by regulating the surface availability of specific guidance receptors/cues (see below).

In addition to interfering with the proper patterning of interneuron axons, AVG ablations also disrupt VNC-associated motor neuron axon guidance. Specifically, the directional outgrowth of D-type motor neuron commissures is perturbed so that these 


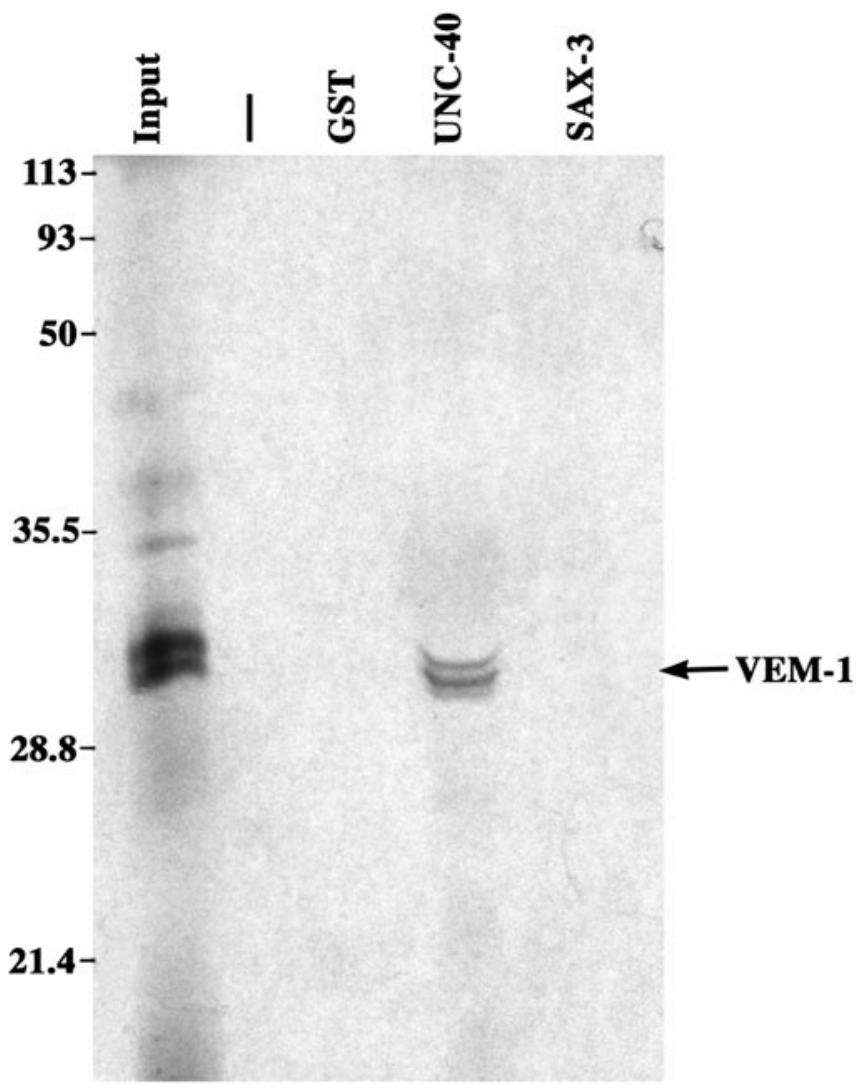

Figure 7. VEM-1 and UNC-40 proteins are capable of associating in vitro. VEM-1 associates in vitro with the cytoplasmic domain of UNC-40 but not with the cytoplasmic domain of SAX-3 or GST alone. The arrow points to the position occupied by the ${ }^{35}$ S-labeled VEM-1 protein probe (runs as a doublet). Input, One-fifteenth of input probe; - , blank lane; GST, GST alone; UNC-40, GST fused to the UNC-40 cytoplasmic domain (amino acids 1106-1415); SAX-3, GST fused to the SAX-3 cytoplasmic domain (amino acids 898-1273). The VEM-1 probe represents the fulllength VEM-1 protein. In all of the cases, molar equivalents of GST fusion proteins were bound to glutathione Sepharose beads and incubated with the ${ }^{35}$ S-labeled VEM-1 protein probe. The beads were then washed, and the bound proteins were subjected to SDS-PAGE (12\%) and autoradiography.

axons inappropriately extend on the left side of the animal (Durbin, 1987; Hutter, 2003). DD and VD motor neuron axons display very similar defects in vem-1 mutants, and, in addition, a subset fails to properly extend within the VNC or along the DV axis. Consistent with an apparent lack of vem-1 expression in motor neurons and the observation that selective expression of vem-1 in AVG rescues motor axon defects (Fig. $3 H$ ), these findings suggest a cell-nonautonomous role for vem-1 in regulating the guidance of motor neuron axons. Thus, it is conceivable that AVG-associated vem-1 regulates both interneuron and motor neuron axon guidance in a cell-nonautonomous manner. That VEM-1 is a membrane protein (Fig. 1) (Runko et al., 1999) does not preclude it from functioning cell nonautonomously. For example, recent studies have shown that Drosophila Kuzbanian has a cell-nonautonomous role in bristle development (Rooke et al., 1996), and, in C. elegans, Eph receptors and ephrin ligands can function cell nonautonomously to regulate epidermal morphogenesis (George et al., 1998; Wang et al., 1999). Furthermore, it appears that the ADAM metalloprotease UNC-71 acts cell nonautonomously through AVG to mediate D-type motor neuron axon guidance (Huang et al., 2003).

\section{vem- 1 is likely to function in the unc-40 pathway}

Previous studies have shown that unc-40 (Hedgecock et al., 1990; Chan et al., 1996; Zallen et al., 1999; Hao et al., 2001; Yu et al., 2002), unc-6 (Hedgecock et al., 1990; Ishii et al., 1992; Wadsworth et al., 1996; Lim et al., 1999), sax-3 (Zallen et al., 1998, 1999; Hao et al., 2001; Yu et al., 2002), slt-1 (Hao et al., 2001), and unc-34 (Yu et al., 2002) are required for the guidance of axons to the VNC and that sax-3 (Zallen et al., 1998, 1999), slt-1 (Hao et al., 2001), unc-34 (Yu et al., 2002), vab-1 (George et al., 1998; Zallen et al., 1999), and nid-1 (Kim and Wadsworth, 2000) play important roles in segregating axons to the right or left tracts of the VNC. In addition, mutations in unc- 6 (Hedgecock et al., 1990; Ishii et al., 1992), unc-5 (Hedgecock et al., 1990; LeungHagesteijn et al., 1992; McIntire et al., 1992), unc-40 (Hedgecock et al., 1990; McIntire et al., 1992; Chan et al., 1996), unc-34 (Colavita and Culotti, 1998), nid-1 (Kim and Wadsworth, 2000), max-1 (Huang et al., 2002), unc-129 (Colavita et al., 1998), unc-71 (Huang et al., 2003), and zag-1 (Clark and Chiu, 2003; Wacker et al., 2003) display vem-1-like, VNC-associated motor neuron axon guidance defects. The results of our double mutant analyses suggest that vem-1 operates in the unc- 40 , but not the sax-3, pathway to regulate the guidance of VNC-associated interneuron and D-type motor neuron axons. However, several observations imply that genetic interactions between vem-1 and unc-40 occur in a cell type-specific context. For example, the loss of vem-1 selectively affects posteriorly (as opposed to anteriorly) projecting interneuron axons. Thus, the posteriorly positioned PVC and DVC interneurons, which extend anteriorly directed axons toward the nerve ring, do not contribute to the aberrant lateral axon phenotype observed in vem-1 mutants. Because PVC axons clearly assume inappropriate lateral positions in unc-40 mutants (Fig. 6D), vem-1 must not cooperate with unc-40 to regulate their pathfinding. Similarly, vem-1 is not required (Fig. 4) for the ventrally directed, unc-40-dependent guidance of axons emanating from specific touch neurons (e.g., AVM) (Chan et al., 1996; Yu et al., 2002). On the other hand, D-type motor neuron commissures inappropriately extend along the left, as opposed to the right, side of the animal in vem-1, but not in unc-40, mutants. Therefore, vem-1 regulates this guidance decision independently of $u n c-40$. The finding that VEM- 1 is capable of binding UNC-40, but not SAX-3, supports our conclusion that vem-1 operates in the unc-40 pathway. Whether a direct interaction between VEM- 1 and UNC-40 is required to mediate particular guidance events, however, remains to be determined.

\section{A possible mechanism for VEM-1 in axon guidance}

Although the primary structure of VEM-1/Vema is predicted to possess a transmembrane domain, it is devoid of motifs that are known to mediate direct cell-cell interactions at the cell surface. However, the cytoplasmic domain of VEM-1/Vema contains several tyrosine-based sorting signals (YXX $\phi$ ) (Fig. 1) that facilitate the rapid internalization or endocytosis of transmembrane proteins from the cell surface to various intracellular compartments in a clathrin-adaptin-dependent manner (Marks et al., 1997; Runko et al., 1999). In the Drosophila VNC, precrossing commissural axons are selectively rendered insensitive to midlineassociated Slit by Commissureless (Tear et al., 1996; Kidd et al., 1998), a sorting facilitator that prevents Robo receptors from reaching the cell surface (Keleman et al., 2002; Myat et al., 2002; Georgiou and Tear, 2003). By analogy with the role that Comm is thought to play at the CNS midline (Keleman et al., 2002; Myat et al., 2002), as well as at the neuromuscular junction (Wolf et al., 1998) in Drosophila, VEM-1 might participate in a rapid remod- 
eling of VNC-associated neurons/axons in C. elegans. Specifically, VEM-1 may facilitate the clearing or presentation of particular guidance receptors/cues along their surfaces (see below). Given that Comm proteins have only been identified in Drosophila (Chisholm and Tessier-Lavigne, 1999; Keleman et al., 2002; Hobert and Bulow, 2003), it is tempting to speculate that VEM$1 /$ Vema might function to regulate the surface availability of key guidance receptors/signals in the C. elegans and vertebrate CNS.

Recent studies have shown that Comm is required in both commissural neurons (Georgiou and Tear, 2002; Keleman et al., 2002) and midline cells (Georgiou and Tear, 2002) for axon guidance across the midline. Similarly, VEM-1 appears to be expressed and function in both posteriorly projecting, $g l r-1:: G F P$-expressing interneurons and the pioneer midline neuron/axon AVG. According to the model proposed above, VEM-1 might function to regulate the surface availability of UNC-40 and/or UNC-6 in ventrally directed follower axons and AVG, respectively. In one possible scenario, VEM-1 may internalize UNC-40 when it is bound to UNC-6. Subsequently, UNC-6 would be expected to dissociate from UNC-40 within intracellular compartments, allowing UNC-40 to be recycled back to the cell surface, where it could participate in another round of UNC6-dependent signaling. In this case, the loss of VEM-1 might result in a net decrease of available UNC-40 receptors at the cell surface. This would compromise the ability of axons to reach the ventral midline and may account for the aberrant lateral axon phenotype displayed by vem-1 mutants. A net decrease of UNC-40 on the surface of AVG should result in a relative excess of free UNC-6, which can lead to defects in the pathfinding of D-type motor neuron axons (Ren et al., 1999). Thus, this type of mechanism might also be consistent with the presumed cellnonautonomous role of AVG-associated vem-1. Future studies will be aimed at determining whether, and under which specific conditions, VEM-1 can mediate the trafficking of VNC neuronaxon-associated guidance receptors and/or their cues.

\section{References}

Altun-Gultekin Z, Andachi Y, Tsalik EL, Pilgrim D, Kohara Y, Hobert O (2001) A regulatory cascade of three homeobox genes, ceh-10, ttx-3 and ceh-23, controls cell fate specification of a defined interneuron class in $C$. elegans. Development 128:1951-1969.

Aurelio O, Hall DH, Hobert O (2002) Immunoglobulin-domain proteins required for maintenance of ventral nerve cord organization. Science 295:686-690.

Brenner S (1974) The genetics of Caenorhabditis elegans. Genetics 77:71-94.

Bulow HE, Boulin T, Hobert O (2004) Differential functions of the C. elegans FGF receptor in axon outgrowth and maintenance of axon position. Neuron 42:367-374.

Chan SS-Y, Zheng H, Su M-W, Wilk R, Killeen MT, Hedgecock EM, Culotti JG (1996) UNC-40, a C. elegans homolog of DCC (deleted in colorectal cancer), is required in motile cells responding to UNC-6 netrin cues. Cell 87:187-195.

Chisholm A, Tessier-Lavigne M (1999) Conservation and divergence of axon guidance mechanisms. Curr Opin Neurobiol 9:603-615

Clark SG, Chiu C (2003) C. elegans ZAG-1, a Zn-finger-homeodomain protein, regulates axonal development and neuronal differentiation. Development 130:3781-3794.

Colavita A, Culotti JG (1998) Suppressors of ectopic UNC-5 growth cone steering identify eight genes involved in axon guidance in Caenorhabditis elegans. Dev Biol 194:72-85.

Colavita A, Krishna S, Zheng H, Padgett RW, Culotti JG (1998) Pioneer axon guidance by unc-129, a C. elegans TGF- $\beta$. Science 281:706-709.

Culotti JG, Merz DC (1998) DCC and netrins. Curr Opin Cell Biol 10:609-613.

Dickson BJ (2002) Molecular mechanisms of axon guidance. Science 298:1959-1964.

Durbin RM (1987) Studies on the development and organization of the nervous system of Caenorhabditis elegans. $\mathrm{PhD}$ thesis, Cambridge University.

Fire A, Xu S, Montgomery MK, Kostas SA, Driver SE, Mello CC (1998) Potent and specific genetic interference by double-stranded RNA in Caenorhabditis elegans. Nature 391:806-811.

George SE, Simokat K, Hardin J, Chisholm AD (1998) The VAB-1 Eph receptor tyrosine kinase functions in neural and epithelial morphogenesis in C. elegans. Cell 92:633-643.

Georgiou M, Tear G (2002) Commissureless is required both in commissural neurons and midline cells for axon guidance across the midline. Development 129:2947-2956.

Georgiou M, Tear G (2003) The N-terminal and transmembrane domains of Commissureless are necessary for its function and trafficking within neurons. Mech Dev 120:1009-1019.

Granato M, Schnabel H, Schnabel R (1994) pha-1, a selectable marker for gene transfer in C. elegans. Nucleic Acids Res 22:1762-1763.

Grunwald IC, Klein R (2002) Axon guidance: receptor complexes and signaling mechanisms. Curr Opin Neurobiol 12:250-259.

Hamelin M, Scott IM, Way JC, Culotti JG (1992) The mec-7 $\beta$-tubulin gene of Caenorhabditis elegans is expressed primarily in touch receptor neurons. EMBO J 11:2885-2893.

Hao JC, Yu TW, Fujisawa K, Culotti JG, Gengyo-Ando K, Mitani S, Moulder G, Barstead R, Tessier-Lavigne M, Bargmann CI (2001) C. elegans Slit acts in midline, dorsal-ventral and anterior-posterior guidance via the SAX-3/Robo receptor. Neuron 32:25-38.

Hart AC, Sims S, Kaplan JM (1995) Synaptic code for sensory modalities revealed by C. elegans GLR-1 glutamate receptor. Nature 378:82-85.

Hedgecock EM, Culotti JG, Hall DH (1990) The unc-5, unc-6, and unc-40 genes guide circumferential migrations of pioneer axons and mesodermal cells on the epidermis in C. elegans. Neuron 2:61-85.

Hein JJ (1990) Unified approach to alignment and phylogenies. Meth Enzymol 183:626-645.

Hobert O, Bulow H (2003) Development and maintenance of neuronal architecture at the ventral midline of C. elegans. Curr Opin Neurobiol 13:1-9.

Hobert O, Moerman DG, Clark KA, Beckerle MC, Ruvkun G (1999) A conserved LIM protein that affects muscular adherens junction integrity and mechanosensory function in Caenorhabditis elegans. J Cell Biol 144:45-57.

Huang X, Cheng H-J, Tessier-Lavigne M, Jin Y (2002) MAX-1, a novel PH/ MyTH4/FERM domain cytoplasmic protein implicated in netrinmediated axon repulsion. Neuron 34:563-576.

Huang X, Huang P, Robinson MK, Stern MJ, Jin Y (2003) UNC-71, a disintegrin and metalloprotease (ADAM) protein regulates motor axon guidance and sex myoblast migration in C. elegans. Development 130:3147-3161.

Hunter CP (2000) Gene silencing: shrinking the black box of RNAi. Curr Biol 10:R137-R140.

Hutter H (2003) Extracellular cues and pioneers act together to guide axons in the ventral cord of C. elegans. Development 130:5307-5318.

Ishii N, Wadsworth WG, Stern BD, Culotti JG (1992) UNC-6, a lamininrelated protein, guides cell and pioneer axon migrations in C. elegans. Neuron 9:873-881.

Jansen G, Hazendonk E, Thijssen KL, Plasterk RH (1997) Reverse genetics by chemical mutagenesis in Caenorhabditis elegans. Nat Genet 17:119-121.

Jin Y, Jorgensen E, Hartwieg E, Horvitz HR (1999) The Caenorhabditis elegans gene unc- 25 encodes glutamic acid decarboxylase and is required for synaptic transmission but not synaptic development. J Neurosci 19:539-548.

Kaprielian Z, Cho K-O, Hadjiargyrou M, Patterson PH (1995) CD9, a major platelet cell surface glycoprotein, is a ROCA antigen and is expressed in the nervous system. J Neurosci 15:562-573.

Kaprielian Z, Runko E, Imondi R (2001) Axon guidance at the midline choice point. Dev Dyn 221:154-181

Keleman K, Rajagopalan S, Cleppien D, Ties D, Paiha K, Huber LA, Technau GM, Dickson BJ (2002) Comm sorts Robo to control axon guidance at the Drosophila midline. Cell 110:415-427.

Kidd T, Russell C, Goodman CS, Tear G (1998) Dosage-sensitive and complementary functions of roundabout and commissureless control axon crossing of the CNS midline. Neuron 20:25-33. 
Kim S, Wadsworth WG (2000) Positioning of longitudinal nerves in C. elegans by nidogen. Science 288:150-154.

Knobel KM, Davis WS, Jorgensen EM, Bastiani MJ (2001) UNC-119 suppresses axon branching in C. elegans. Development 128:4079-4092.

Leung-Hagesteijn C, Spence AM, Stern BD, Zhou Y, Su MW, Hedgecock EM, Culotti JG (1992) UNC-5, a transmembrane protein with immunoglobulin and thrombospondin type 1 domains, guides cell and pioneer axon migrations in C. elegans. Cell 71:289-299.

Lim Y-S, Mallapur S, Kao G, Ren X-C, Wadsworth WG (1999) Netrin UNC- 6 and the regulation of branching and extension of motoneuron axons from the ventral nerve cord of Caenorhabditis elegans. J Neurosci 19:7048-7056.

Maduro M, Pilgrim D (1995) Identification and cloning of unc-119, a gene expressed in the Caenorhabditis elegans nervous system. Genetics 141:977-988.

Maricq AV, Peckol E, Driscoll M, Bargmann CI (1995) Mechanosensory signalling in C. elegans mediated by the GLR-1 glutamate receptor. Nature 378:78-81.

Marks MS, Ohno H, Kirchhausen T, Bonifacino JS (1997) Protein sorting by tyrosine-based signals: adapting to the Ys and wherefores. Trends Cell Biol 7:124-128.

McIntire SL, Garriga G, White J, Jacobson D, Horvitz HR (1992) Genes necessary for directed axonal elongation or fasciculation in C. elegans. Neuron 8:307-322.

Mello CC, Fire A (1995) DNA Transformation. In: Caenorhabditis elegans: modern biological analysis of an organism (Epstein HF, Shakes DC, eds), pp 451-482. San Diego: Academic.

Miller DM, Niemeyer CJ (1995) Expression of the unc-4 homeoprotein in Caenorhabditis elegans motor neurons specifies presynaptic input. Development 121:2877-2886.

Montgomery MK, Fire A (1998) Double-stranded RNA as a mediator in sequence-specific genetic silencing and co-suppression. Trends Genet 14:255-258.

Montgomery MK, Xu S, Fire A (1998) RNA as a target of double-stranded RNA-mediated genetic interference in Caenorhabditis elegans. Proc Natl Acad Sci USA 95:15502-15507.

Myat A, Henry P, McCabe V, Flintoft L, Rotin D, Tear G (2002) Drosophila Nedd4, a ubiquitin ligase, is recruited by commissureless to control cell surface levels of the roundabout receptor. Neuron 35:447-459.

Reddien P, Cameron S, Horvitz H (2001) Phagocytosis promotes programmed cell death in C. elegans. Nature 412:198-202.

Ren XC, Kim S, Fox E, Hedgecock EM, Wadsworth WG (1999) Role of netrin UNC-6 in patterning the longitudinal nerves of Caenorhabditis elegans. J Neurobiol 39:107-118.

Rooke J, Pan D, Xu T, Rubin GM (1996) KUZ, a conserved metalloproteasedisintegrin protein with two roles in Drosophila neurogenesis. Science 273:1227-1231.

Runko E, Kaprielian Z (2002) Expression of Vema in the developing mouse spinal cord and optic chiasm. J Comp Neurol 451:289-299.

Runko E, Wideman C, Kaprielian Z (1999) Cloning and expression of VEMA: a novel ventral midline antigen in the rat CNS. Mol Cell Neurosci 14:428-443.

Stein E, Tessier-Lavigne M (2001) Hierarchial organization of guidance receptors: silencing of netrin attraction by Slit through a Robo/DCC receptor complex. Science 291:1928-1938.
Tavernarakis N, Wang S-L, Dorovkov M, Ryazanov A, Driscoll M (2000) Heritable and inducible genetic interference by double-stranded RNA encoded by transgenes. Nat Genet 24:180-183.

Tear G, Harris R, Sutaria S, Kilomanski K, Goodman CS, Seeger MA (1996) Commissureless controls growth cone guidance across the CNS midline in Drosophila and encodes a novel membrane protein. Neuron 16:501-514.

Tessier-Lavigne M, Goodman CS (1996) The molecular biology of axon guidance. Science 274:1123-1133.

Troemel ER, Chou JH, Dwyer ND, Colbert HA, Bargmann CI (1995) Divergent seven transmembrane receptors are candidate chemosensory receptors in C. elegans. Cell 83:207-218.

Tsalik EL, Niacaris T, Wenick AS, Pau K, Avery L, Hobert O (2003) LIM homeobox gene-dependent expression of biogenic amine receptors in restricted regions of the C. elegans nervous system. Dev Biol 263:81-102.

Wacker I, Schwarz V, Hedgecock EM, Hutter H (2003) zag-1, a Zn-finger homeodomain transcription factor controlling neuronal differentiation and axon outgrowth in C. elegans. Development 130:3795-3805.

Wadsworth WG (2002) Moving around in a worm: netrin UNC-6 and circumferential axon guidance in C. elegans. Trends Neurosci 25:423-429.

Wadsworth WG, Bhatt H, Hedgecock EM (1996) Neuroglia and pioneer neurons express UNC-6 to provide global and local netrin cues for guiding migrations in C. elegans. Neuron 16:35-46.

Wang X, Roy PJ, Holland SJ, Zhang LW, Culotti JG, Pawson T (1999) Multiple ephrins control cell organization in C. elegans using kinasedependent and -independent functions of the Vab-1 Eph receptor. Mol Cell 4:903-913.

White JG, Southgate E, Thomson JN, Brenner S (1976) The structure of the ventral nerve cord of Caenorhabditis elegans. Philos Trans R Soc Lond B Biol Sci 275:327-348.

White JG, Southgate E, Thomson JN, Brenner S (1986) The structure of the nervous system of the nematode Caenorhabditis elegans. Philos Trans R Soc Lond B Biol Sci 314:1-340.

Wightman B, Baran R, Garriga G (1997) Genes that guide growth cones along the C. elegans ventral nerve cord. Development 124:2571-2580.

Wolf B, Seeger MA, Chiba A (1998) Commissureless endocytosis is correlated with initiation of neuromuscular synaptogenesis. Development 125:3853-3863

Yu TW, Bargmann CI (2001) Dynamic regulation of axon guidance. Nat Neurosci 4:1169-1176.

Yu TW, Hao JC, Lim W, Tessier-Lavigne M, Bargmann CI (2002) Shared receptors in axon guidance: SAX-3/Robo signals via UNC-34/Enabled and a netrin-independent UNC-40/DCC function. Nat Neurosci 5:1147-1154.

Zallen JA, Yi BA, Bargmann CI (1998) The conserved immunoglobulin superfamily member SAX-3/Robo directs multiple aspects of axon guidance in C. elegans. Cell 92:217-227.

Zallen JA, Kirch SA, Bargmann CI (1999) Genes required for axon pathfinding and extension in the $C$. elegans nerve ring. Development 126:3679-3692.

Zhu Q, Runko E, Imondi R, Milligan T, Kapitula D, Kaprielian Z (1998) New cell surface marker of the rat floor plate and notochord. Dev Dyn 211:314-326. 